\title{
Multistability and high reflectance of a mono- layer of three-level quantum emitters with a doublet in the excited state
}

\author{
Davut Bayramdurdiyev 1,*, Ramil Malikov ${ }^{1}$, Igor Ryzhov², and Victor Malyshev ${ }^{2,3}$ \\ ${ }^{1}$ Akmullah State Pedagogical University of Bashkortostan, 450000 Ufa, Russia \\ ${ }^{2}$ Hertsen State Pedagogical University of Russia, 191186 St.-Petersburg, Russia \\ ${ }^{3}$ ZIAM, University of Groningen, Nijenborgh 4, 9747 AG Groningen, The Netherlands
}

\begin{abstract}
We study theoretically the nonlinear optical response of a mono-layer of three-level quantum emitters with a doublet in the excited state. It is shown that the layer's response exhibits multistability. In a certain frequency range, the monolayer operates as a perfect bistable mirror.
\end{abstract}

We conduct a theoretical study of the steady-state optical response of a monolayer of regularly spaced three-level quantum emitters (QEs) with a doublet in the excited state. The total (retarded) dipole-dipole interaction of QEs is taken into account. This interaction provides a positive feedback. The interplay of the latter and the immanent nonlinearity of QE's gives rise to a multistability of the monolayer optical response. In a certain frequency range, the system operates as a nanometric bistable mirror.

It is assumed that the monolayer undergoes an action of a CW external field of a Rabi amplitude $\Omega_{0}$ and frequency $\omega_{0}$, which is quasi-resonant with the QE's allowed transitions. A constituent QE is modelled by a three-level V-type quantum system with the ground state $|1\rangle$, and a doublet $|2\rangle$ and $|3\rangle$ in the excited states. The allowed optical transitions are $|1\rangle \leftrightarrow|2\rangle$ and $|1\rangle \leftrightarrow|3\rangle$. They are characterized by the transition dipole moments $\mathrm{d}_{21}$ and $\mathrm{d}_{31}$, transition frequencies $\omega_{21}$ and $\omega_{31}$, and spontaneous decay constants $\gamma_{21}$ and $\gamma_{31}$. The doublet is described by the splitting $\Delta_{32}$ and the relaxation constant $\gamma_{32}$.

The optical dynamics of a constituent QE is governed by the $3 \times 3$ density matrix $\rho_{\alpha \beta}(\alpha, \beta$ $=1,2,3)$. The total field $\Omega$ acting on a given $\mathrm{QE}$ in the monolayer represents a sum of the external field $\Omega_{0}$ and the field produced by all others QEs in place of the given one. In this way, the total (retarded) QE-QE dipole-dipole interaction is taken into account. The nearzone (far-zone) part of the QE-QE interaction gives rise to a dynamic renormalization of the transition frequencies $\omega_{21}$ and $\omega_{32}$ (relaxation constants $\gamma_{21}$ and $\gamma_{31}$ ), depending on the population difference of corresponding transitions [1,2]. The effects are described by the constants $\Delta_{\mathrm{L}}$ (shift) and $\gamma_{\mathrm{R}}$ (relaxation). These parameters govern a positive feedback which is responsible for a sophisticated nonlinear optical properties of the monolayer.

In Fig. 1, we present the results of the steady-state calculations performed for the case when the external field is tuned into the resonance with the transition $|1\rangle \leftrightarrow|3\rangle\left(\Delta_{31}=\omega_{31}-\right.$

${ }^{*}$ Corresponding author: d.bayramdurdiyev@gmail.com 
$\omega_{0}=0$ ). Panel (b) shows the acting field magnitude $|\Omega|$ as a function of the external field magnitude $\left|\Omega_{0}\right|$ for the set of parameters typical for two-dimensional supercrystals built up of semiconductor quantum dots (SQD) [3]. As is seen from the plot, $|\Omega|$ appears to be a multi-valued function of $\left|\Omega_{0}\right|$, signaling multistability. The stability of different parts of the steady-state solution has been checked by analyzing the spectrum of Lyapunov exponents $\Lambda_{\mathrm{k}}(\mathrm{k}=1,2 \ldots 8)$. The maximal real part of $\left\{\Lambda_{\mathrm{k}}\right\}, \operatorname{Max}\{\operatorname{Re}[\Lambda]\}$, is plotted in panel (c).

(a)

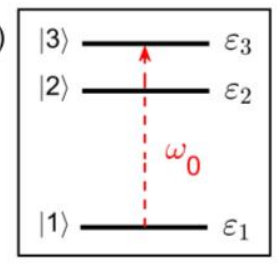

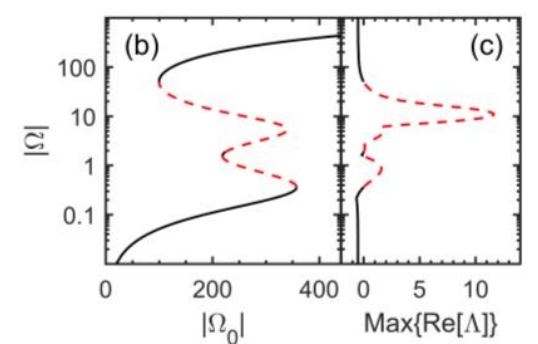

Fig. 1. (a) - Excitation scheme of a $Q E\left(\Delta_{31}=0\right)$. (b) - Steady-state solution for $|\Omega|$ as function of $\left|\Omega_{0}\right|$. Solid (dashed) parts of the curves indicate stable (unstable) regions of $|\Omega|$. (c) - Real part of the major Lyapunov exponent $\operatorname{Max}\{\operatorname{Re}[\Lambda]\}$ as a function of $|\Omega|$. Parameters of calculations are: $\Delta_{32}=10$, $\gamma_{\mathrm{R}}=100, \Delta_{\mathrm{L}}=1000, \gamma_{32}=0.01$. All quantities is given in units of $\gamma_{31}$.
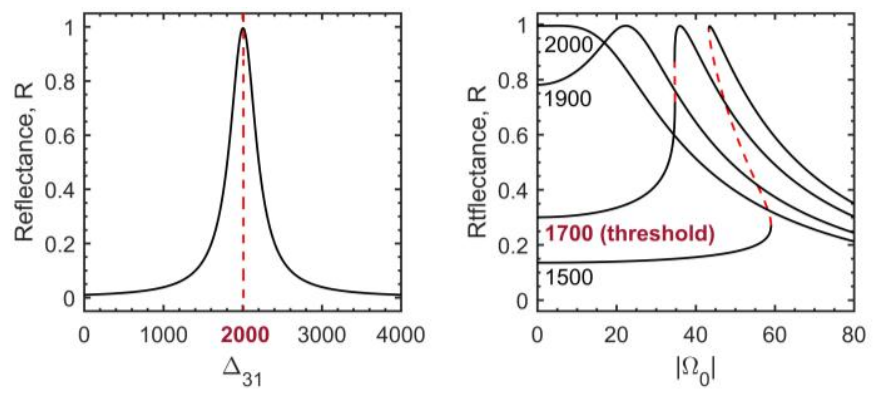

Fig. 2. Left panel - the linear reflection coefficient $R$ as a function of the detuning $\Delta_{31}$. Right panel the field dependence of $R$ computed for a set of detunings $\Delta_{31}$ shown in the plot. The value $\Delta_{31}=1700$ is the threshold for bistability to occur. The rest of parameters are the same as in Fig. 1.

Fig. 2 shows the detuning and field dependence of the reflectance $\mathrm{R}=\left|\Omega_{\text {ref }} / \Omega_{0}\right|^{2}$ (left and right panels, respectively), $\Omega_{\text {refl }}=\gamma_{\mathrm{R}}\left(\rho_{31}+\rho_{21}\right)$ is the Rabi amplitude of the reflected field. As follows from the left plot, the linear reflectance (for a week $\left|\Omega_{0}\right|$, left panel) has a maximum at $\Delta_{31}=2000 \gamma_{31}$. Moreover, at this point $\mathrm{R}$ is approaches unity, i.e. the monolayer almost totally reflects the input field. The right panel in Fig. 2 demonstrates arising three solutions for $\mathrm{R}$ at a given $\left|\Omega_{0}\right|$, which means bistability of the reflectance.

Summarizing, we believe that a monolayer comprising V-type QEs may serve as a nanometric bistable mirror. These features might be of interest for nanophotonics. Supercrystals built up of SQDs with the degenerate valence band, e.g. CdSe, placed in magnetic field [4], can be considered as candidates for realization of such systems.

D.Y.B and R.F.M. acknowledge Akmullah State Pedagogical University for a support.

\section{References}

1. R.F. Malikov, V.A. Malyshev, Opt Spectrosc. 122, 955 (2017)

2. V.A. Malyshev et al., J. Phys.: Conf. Ser. 1220, 012006 (2019)

3. M.P. Boneschanscher, W.H. Evers, J.J. Geuchies et al., Science 344, 1377 (2014)

4. Al.L. Efros, M. Rosen, M. Kuno et al., Phys. Rev. B. 54, 4843 (1996) 